\title{
Evolution of neuro-endovascular surgery from serendipity and sagacity to evidence based medicine, history and future directions
}

\section{Editorial}

The most common disorders affecting the cerebral circulation are strokes. Stroke is one of the leading causes of permanent disability, and is the third largest cause of death in western countries. Annually, more than 700,000 individuals in the United States experience new or recurrent strokes ${ }^{1}$. If this number is correlated to the size of the international population, the predicted number worldwide will yield a huge sum of cases, with its huge burden on the health care systems, families, and the whole economy.

The etiology of stroke includes both cerebral hemorrhage originated for example from cerebral aneurysm or brain AVM and ischemic cerebral infarction results from occlusive disease of the extra cranial or major intracranial arteries (atherosclerotic lesions).

The etiology of stroke includes both cerebral hemorrhage originated for example from cerebral aneurysm or brain AVM and ischemic cerebral infarction results from occlusive disease of the extra cranial or major intracranial arteries (atherosclerotic lesions).

The history of neuro-endovascular therapy starts with the work done by Egas Moniz in 1926. ${ }^{2,3}$ Egas Moniz was the first to perform a complete cerebral angiogram that included arterial and venous phases; he was inspired by the use of iodized contrast oil in myelography. ${ }^{2,3}$ Cerebral angiography became an important diagnostic tool since then and was done with percutaneous carotid puncture technique.,

The two most important revolutionary steps in this field of endovascular neurosurgery came from the serendipitous observation by Serbinenko's of a child's helium-filled balloon leading him to the concept of detachable balloons for vessel sacrifice and Guglielmi's sagacity which is due to his penetrating intelligence and keen perception of observing accidental electrolytic detachment of a steel electrode when trying to induce Aneurysmal electro-thrombosis leading to the development of the detachable coil. ${ }^{1}$

In the past decade cerebral endovascular approaches has evolved rapidly through advances in tools and techniques and as a result of the more growing need for evidence based medicine. More patients with diseases traditionally treated via surgical techniques can now be treated with techniques that focus on endo-luminal reconstruction. ${ }^{1}$ In the future the role of personalized or individualized medicine will increase. Better diagnoses, earlier interventions, more-efficient drug therapies, and customized treatment plans will be the promises of
Volume I Issue I - 2014

\author{
Waseem Aziz \\ Consultant of Neuroendovascular and Neurosurgery, Alexandria \\ University, Egypt \\ Correspondence: : Waseem Aziz, Consultant of \\ Neuroendovascular and Neurosurgery, Alexandria University, \\ Egypt, 9 Farouk Darwish St. Sidi Bishr Bahry,Alexandria, Egypt, Tel \\ 020II55544166, Email wasim.aziz@wasim.aziz
}

Received: May 0I, 2014 | Published: May 02, 2014

personalized medicine. We will continue to depend on serendipity and sagacity of smart people to revolutionize our field from time to time. Stroke will offer the largest growth area of endovascular intervention and investing in dedicated stroke units will provide superior care as measured by survival rate, functional outcome, and proportion of patients returning home.

\section{Acknowledgments}

None.

\section{Conflicts of interest}

The authors of the manuscript of reference state that there is no conflict of interest related to the article.

\section{References}

1. Hopkins LN, Ecker RD. Cerebral endovascular neurosurgery. Neurosurgery. 2008;62(6 Suppl 3):1483-1501.

2. Richling B. History of endovascular surgery: personal accounts of the evolution. Neurosurgery. 2006;59(5 Suppl 3):S30-S38.

3. Hopkins LN, Lanzino G, Guterman LR. Treating complex nervous system vascular disorders through a "needle stick": origins, evolution, and future of neuroendovascular therapy. Neurosurgery. 2001;48(3):463-475.

4. Prestigiacomo CJ. Historical perspectives: the microsurgical and endovascular treatment of aneurysms. Neurosurgery. 2006;59(5Suppl 3):S39-S47. 